\title{
Assessment of burned coal shale properties based on cyclic load
}

\author{
Marcin Grygierek ${ }^{1, *}$, Piotr Kalisz ${ }^{2}$, Krzysztof Pacześniowski ${ }^{2}$, Andrzej Pytlik ${ }^{2}$, Magdalena \\ Zięba $a^{2}$ \\ ${ }^{1}$ Silesian University of Technology, Faculty of Civil Engineering, Gliwice, Poland \\ ${ }^{2}$ Central Mining Institute, Katowice, Poland
}

\begin{abstract}
Road surfaces that are subjected to cyclic loads generated by vehicle wheels must meet the requirements concerning the durability in the assumed period of use. The durability of the layered pavement construction systems depends on the value and frequency of the load as well as on the mechanical features of its individual layers. Layers of unbound, mechanically stabilized mixtures are a significant aspect of surfaces that are susceptible. Mixtures of this type can be applied both to the subgrade layers as well as to the bottom pavement layers, including the improved course. Considering the cyclic nature of the load on the surface of the entire system, mechanically stabilized layers are subject to continuous, but slow, densification during the period of use, which results in the formation of permanent deformations and so-called structural ruts. Post-mining waste is frequently used in road construction. which is the so-called burned shale that can be used for the bottom layers of the surface and layers of the improved subgrade (soil replacement). This material was the subject of the analysis. The evaluation was based mainly on the results of pilot studies covering cyclic loads of the layer/course made of the so-called red shale. The applied research method was aimed at preliminary assessment of its suitability for the assessment of the behaviour of the disintegrated medium under the conditions of test loads simulating the movement of vehicles.
\end{abstract}

Keywords: static and cyclic plate load testing, burned coal shale

\section{Introduction}

Road surface constructions usually use layers of mechanically stabilized aggregate which is not chemically bonded. These layers can be applied in the lower layer of the subgrade and/or in the lower layers of the surface and in the improved course. Mainly these layers and ground course are responsible for the development of the so-called structural deformations that are observed in the wheel tracks. Structural deformations are also referred to as so-called structural ruts. Their origin is related to the densification of the granular

* Corresponding author: marcin.grygierek@polsl.pl 
medium influenced by cyclic loading of layers generated by heavy vehicles. The evaluation of the properties of layers made of mechanically stabilized material is based on the evaluation of the modulus that a given material can achieve and the deformation as a function of the number of loads [1]. The assessment of the mechanical properties of aggregates, including the assessment of their non-linear behaviour, is the subject of many studies and analyses $[2,3,4,5,6]$. The value of the modulus is determined by several factors, including grain size of the mixture, its moisture, density of the layer prior observation, condition of the subgrade (its moisture content), deformations occurring in mining areas. Previous research and observations indicate that the modulus of the unbound layer can be further increased even still in the course of using the pavement, although this depends on the conditions occurring in subgrade of the analysed layer.

The article presents the results of pilot laboratory tests of aggregate subjected to cyclic loads, simulating the operation of aggregates during the use of the pavement. The research involved the use of burned shale, which is a waste created during the mining of hard coal. This material is applied to all layers of earthworks [7] to lower layers of pavement construction and layers of the improved subgrade.

\section{Characteristic of burned coal shale}

The use of waste materials in construction, especially communication systems, is extremely important in terms of the possibility of development, which reduces the problems associated with their storage and environmental impact [3]. Current rich experience obtained thanks to the use of waste accompanying mining of hard coal shows that burned coal shale, referred to as splinter shale, show significantly better properties than not burned coal shales. The predominance of burned shales results mainly from better resistance to water and frost and lower susceptibility to fragmentation during their compaction (Fig. 1).

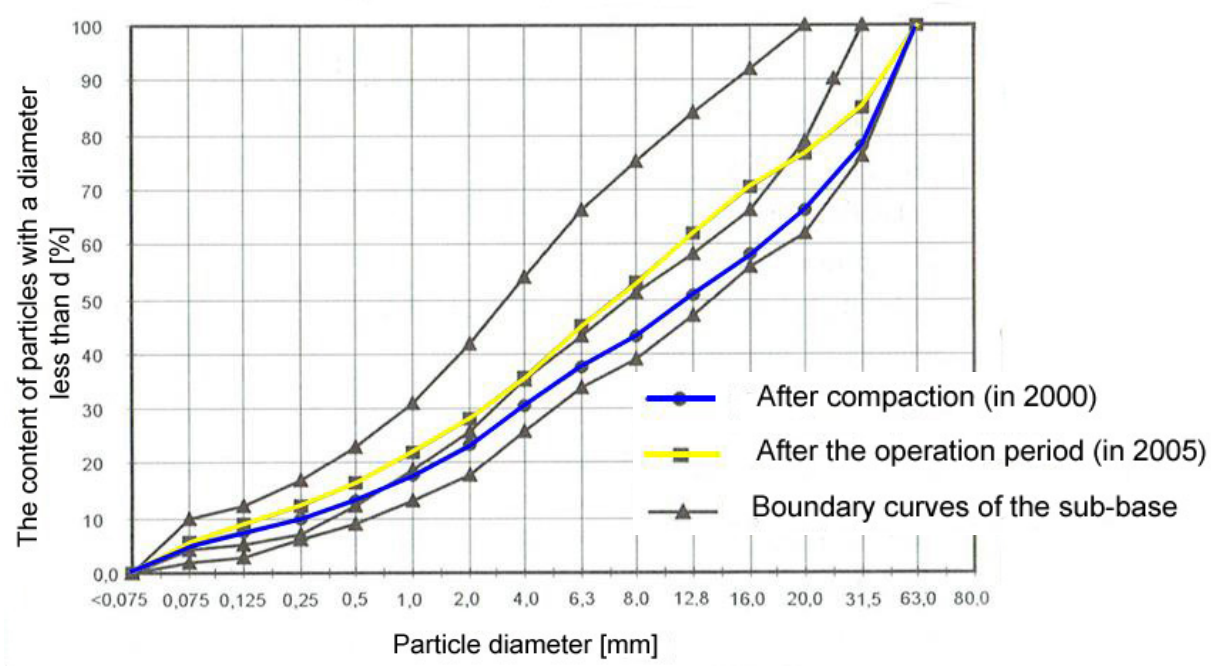

Fig. 1. Granularity of aggregate made of burned coal shale after compacting and five years of road use [8]

As Rafalski points out [8], burned coal shale can be used for road foundations and other lower layers. Rafalski [8] is one of the many researchers who presented the field experiments describing the behaviour of burned coal shale (Fig. 1) applied in a road. These works present such aspects as deformation moduli, determined on the basis of a static VSS 
test, in different periods of surface use, i.e. 0 - 5 years (Tab. 1) - after 5 years, it was observed that the parameters increased from $128 \mathrm{MPa}$ to $142 \mathrm{MPa}$.

Table 1. Results of tests on deformation moduli of burned coal shale in road pavement [8]

\begin{tabular}{|c|c|c|}
\hline Period of use & $\begin{array}{c}\text { Secondary deformation modulus } \\
\mathbf{E}_{\mathbf{2}}[\mathbf{M P a}]\end{array}$ & $\begin{array}{c}\text { Deformation index } \\
\mathbf{I}_{\mathbf{0}}\end{array}$ \\
\hline \hline 0 months & 128 & 2.0 \\
\hline 3 months & 129 & 2.0 \\
\hline 6 months & 136 & 1.9 \\
\hline 5 years & 142 & 2.0 \\
\hline
\end{tabular}

\section{Characteristics of the testing station}

The tests of cyclic loading of aggregate were carried out in laboratory conditions, in a box with a cross-section shown in Figure 3 and a length of $1.20 \mathrm{~m}$. The testing station was placed on a concrete stand, and the side walls were made of $2.5 \mathrm{~cm}$ thick steel sheet, which was additionally ribbed. An integral element of the station is the frame, which is a counterbalance to the hydraulic cylinder exerting a load on the surface of the layer system under test. Two layers of material were built in the box (Fig. 3).

The graining of the sand used indicates that it is a coarse sand with a uniformity coefficient $\mathrm{Cu}=2.2$, which classifies this sand as poorly graded (uniformly-graded) soil $(\mathrm{Cu}<5)$, hardly compactable. The grain size of the shale was compared with the required field of proper graining for individual pavement layers according to Technical Requirements for Unbound Mixtures [6], as shown in Figure 2.
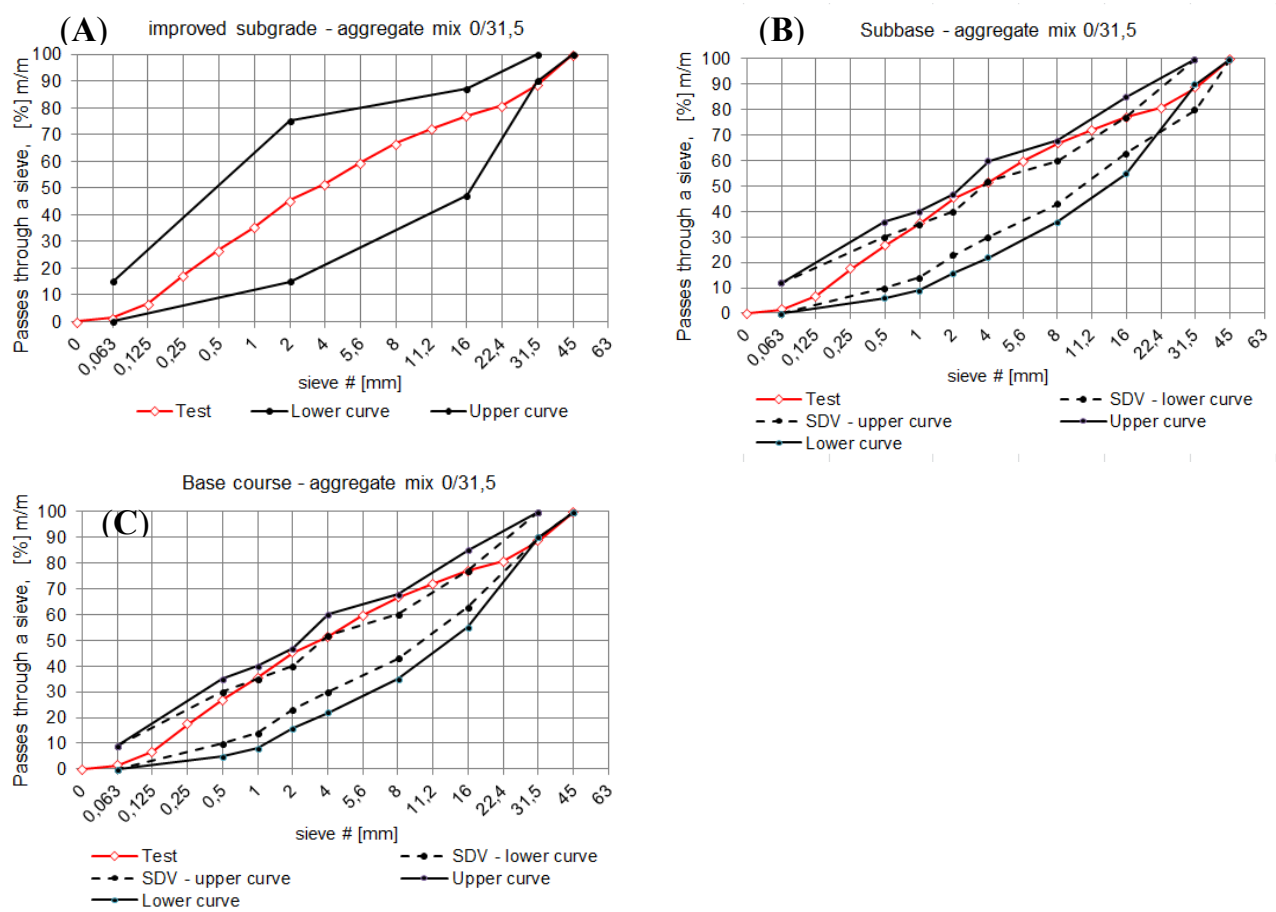

Fig. 2. Comparison of the graining curve of the tested mixture with proper graining curves [6]

The analysis of the course of the grain curve of the shale used clearly indicates that course is close to the upper limit of proper graining. In particular, it applies to areas of proper 
graining for subbase and subgrade. A comparison of the test results of selected aggregate properties and their comparison with the requirements for individual layers is presented in Table 2.

Table 2. Comparison of selected properties of burned coal shale and sand according to [9] and [6]

\begin{tabular}{|c|c|c|c|c|c|c|c|c|c|}
\hline \multirow{3}{*}{ 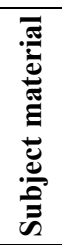 } & & & \multirow{3}{*}{$\begin{array}{c}\text { Test } \\
\text { results }\end{array}$} & \multicolumn{6}{|c|}{ Requirements } \\
\hline & \multirow{2}{*}{\multicolumn{2}{|c|}{$\begin{array}{c}\text { Subject } \\
\text { parameters }\end{array}$}} & & \multicolumn{2}{|c|}{ Base course } & \multirow{2}{*}{$\begin{array}{c}\text { Subbase } \\
\text { KR3- } \\
\text { KR7 } \\
\end{array}$} & \multicolumn{2}{|c|}{$\begin{array}{c}\text { Frost } \\
\text { protection } \\
\text { layer }\end{array}$} & \multirow{2}{*}{$\begin{array}{c}\begin{array}{c}\text { Layer of } \\
\text { improved } \\
\text { subgrade }\end{array} \\
\text { KR1- } \\
\text { KR7 } \\
\end{array}$} \\
\hline & & & & $\begin{array}{l}\text { KR1- } \\
\text { KR2 }\end{array}$ & $\begin{array}{l}\text { KR3- } \\
\text { KR7 }\end{array}$ & & $\begin{array}{l}\text { KR1- } \\
\text { KR2 }\end{array}$ & $\begin{array}{l}\text { KR3- } \\
\text { KR7 }\end{array}$ & \\
\hline \multirow{3}{*}{ 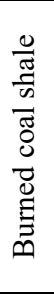 } & $\begin{array}{l}\text { Sand } \\
\text { index }\end{array}$ & {$[\%]$} & 43.05 & $\geq 45$ & $\geq 45$ & $\geq 40$ & $\geq 35$ & $\geq 35$ & $\geq 35$ \\
\hline & $\begin{array}{l}\text { CBR } \\
\text { index }\end{array}$ & {$[\%]$} & 57.2 & $\geq 60$ & $\geq 80$ & $\geq 60$ & $\geq 25$ & $\geq 35$ & $\geq 20$ \\
\hline & \multicolumn{2}{|c|}{$\begin{array}{l}\text { Compatibility of } \\
\text { the grain size with } \\
\text { a proper graining } \\
\text { field }\end{array}$} & Fig. 2 & $\begin{array}{l}\left.\text { yes }^{*}\right) \\
0 / 31.5\end{array}$ & $\begin{array}{l}\left.\text { yes }^{*}\right) \\
0 / 31.5\end{array}$ & $\begin{array}{l}\left.\text { yes }^{*}\right) \\
0 / 31.5\end{array}$ & $\begin{array}{c}\left.\text { yes }^{*}\right) \\
0 / 31.5\end{array}$ & $\begin{array}{c}\left.\text { yes }^{*}\right) \\
0 / 31.5\end{array}$ & $\begin{array}{l}\left.\text { yes }^{*}\right) \\
0 / 31.5\end{array}$ \\
\hline
\end{tabular}

*) graining curve close to the limit curve

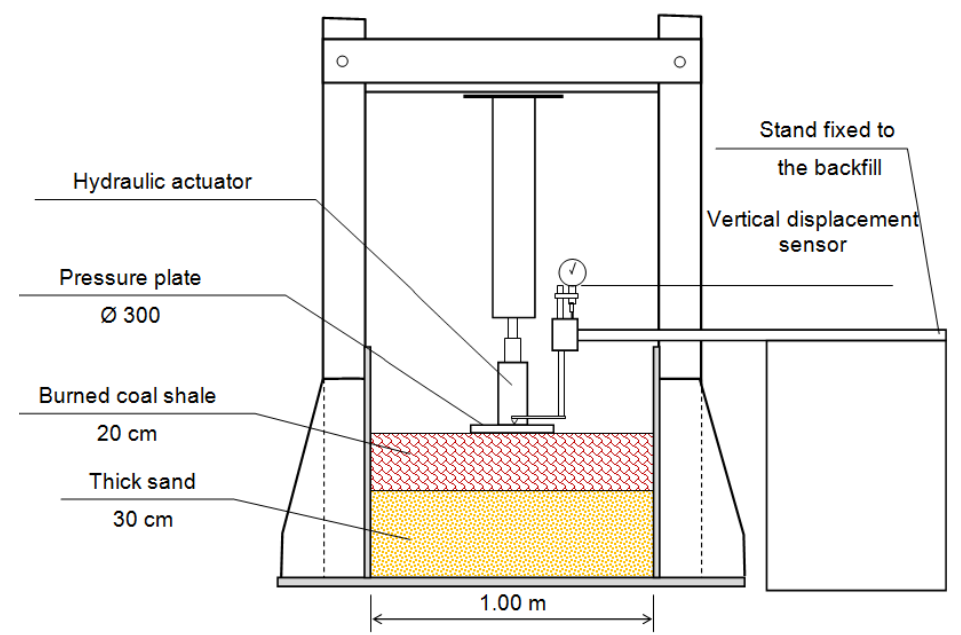

Fig. 3. Scheme of the testing station
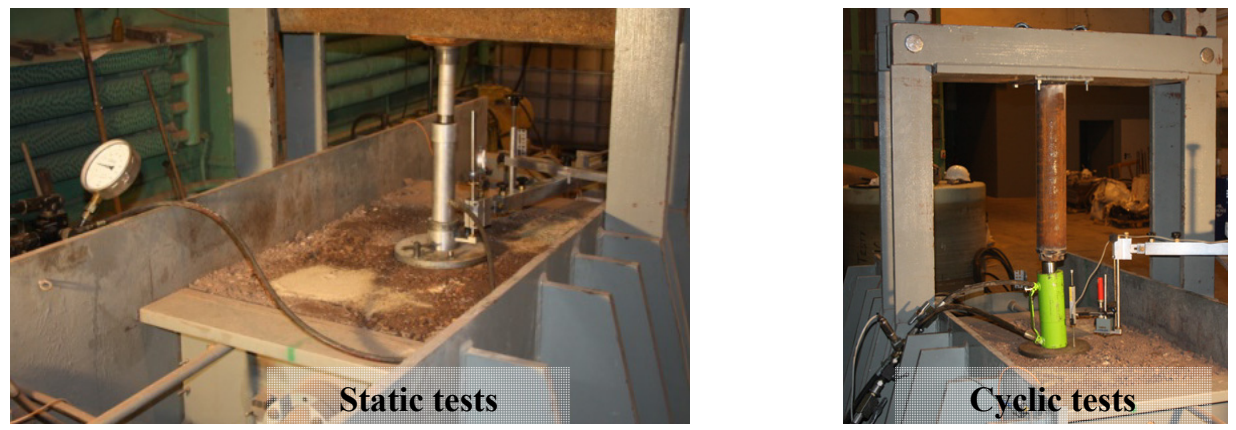

Fig. 4. The testing station during static and cyclic loading of aggregate

The static tests used the so-called VSS plate - i.e. a steel plate with a diameter of $30 \mathrm{~cm}$, a hydraulic actuator and a stand for mounting the displacement sensor on a steel plate (Fig.4). 
The tests based on cyclic loading of the aggregate layer were carried out by loading the surface of the system, also with a steel plate with a diameter of $30 \mathrm{~cm}$, whereby the hydraulic actuator was controlled using a hydraulic pump and a control programme (Fig.4).

\section{Implementation and tests}

The tests were carried out in three stages. In the first stage, a static VSS plate measurement was made on the surface of the layer system (Fig. 5), marking the primary and secondary deformation moduli. In the second stage, the layer system was subjected to cyclic loading, using a hydraulic actuator, a hydraulic pump and a control programme. A load of $550 \mathrm{kPa}$ with a frequency of $0.5 \mathrm{~Hz}$ was applied to the surface of the layers. In total, 430 load cycles were carried out (Fig. 7). The tests covered measurements of vertical displacements of the plate and the value of the force transmitted to the plate at a frequency of $25 \mathrm{~Hz}$. The third stage of the research included the re-execution of the static measurement with the VSS plate (Fig. 6), which was used to calculate the primary and secondary deformation modulus. The final pressure was taken equal to that used during the cyclic test, i.e. $550 \mathrm{kPa}$.
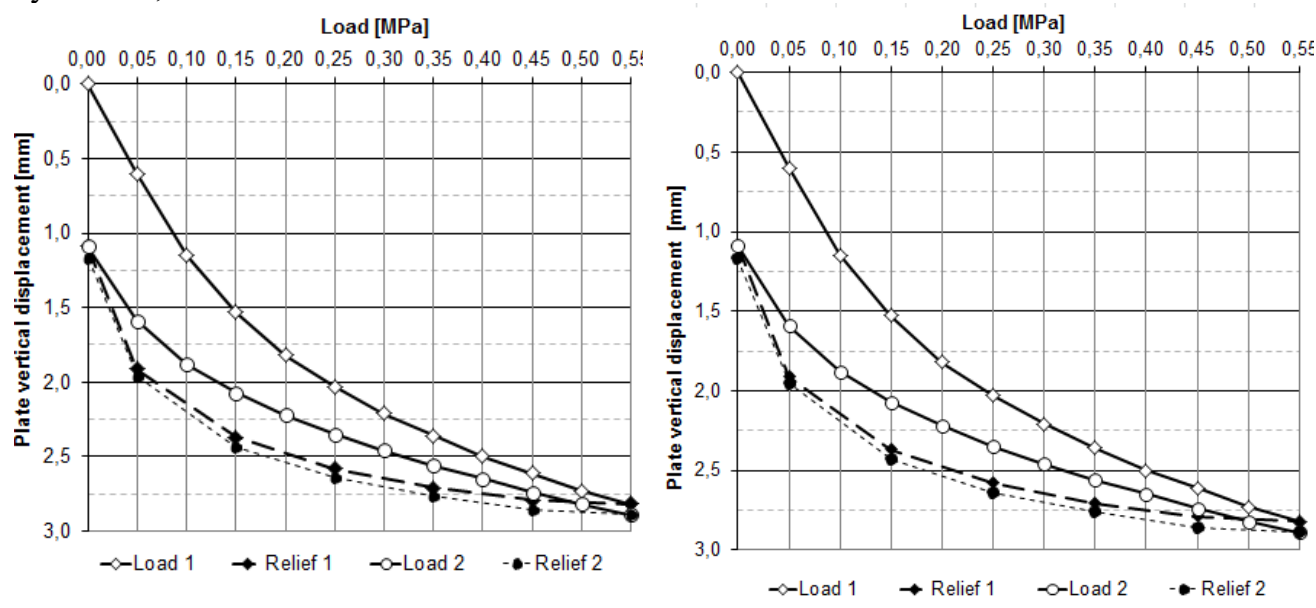

Fig. 5. Results of the static VSS plate measurement before the cyclic tests commence

Fig. 6. Results of measurement with a static VSS plate after cyclic tests

The primary and secondary deformation moduli in the first and third stages were calculated as for the stress range of $q=150-250 \mathrm{kPa}$ (Tab. 3).

Table 3. Calculated results of deformation moduli based on static tests (VSS plate)

\begin{tabular}{|l|c|c|c|}
\hline \multirow{2}{*}{ Research stage } & \multicolumn{2}{|c|}{ Deformation modulus } & Deformation indicator \\
\cline { 2 - 4 } & $\mathbf{E}_{\mathbf{1}}[\mathbf{M P a}]$ & $\mathbf{E}_{\mathbf{2}}[\mathbf{M 0 P a}]$ & $\mathbf{I}_{\mathbf{0}}=\mathbf{E}_{\mathbf{2}}$ / $\mathbf{E}_{\mathbf{1}}$ \\
\hline \hline Prior the cyclic loading test & 36.3 & 75 & 2.06 \\
\hline After the cyclic loading test & 45.0 & 80 & 1.78 \\
\hline
\end{tabular}

In the second stage (cyclic test) the modulus was calculated in each load cycle according to the relationship (1). The results are shown in Figure 7.

$$
E_{r N}=\omega \frac{\Delta q D}{\Delta s_{N}}
$$


where:

$\omega \quad-$ impact factor, for circular load surface $\omega=1.0$,

$\Delta q-$ surface load increase [ $\mathrm{kPa}]$,

$D$ - diameter of the circular loading surface [m],

$\Delta s_{N}-$ increase of surface displacement [mm].

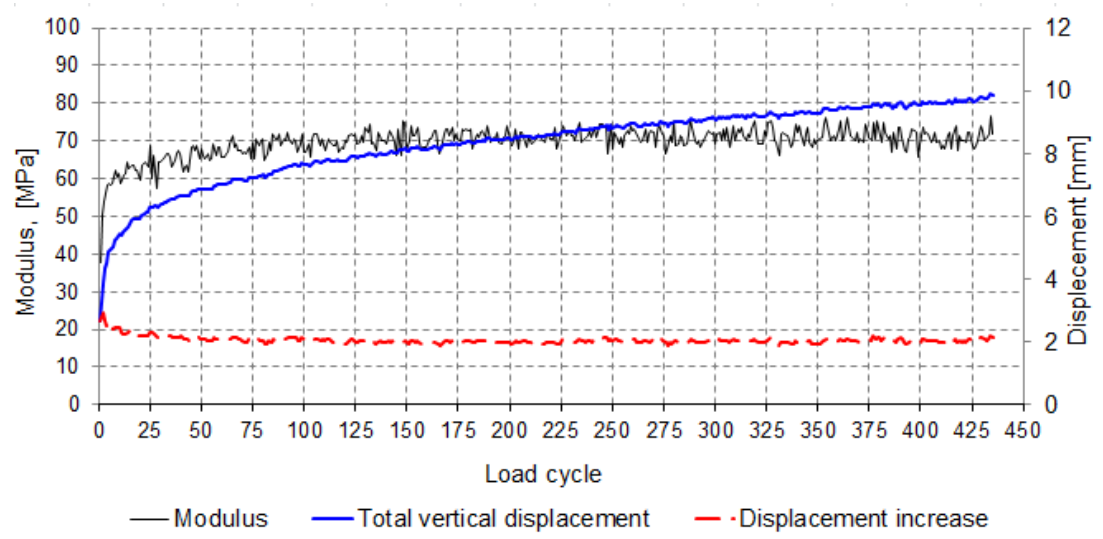

Fig. 7. Results of cyclic tests

\section{Analysis and discussion of the results}

The tested layer system before the cyclic test was characterized by a strain index $\mathrm{I}_{0}=2.06$. This result indicates good density $\left(\mathrm{I}_{0} \leq 2.2\right)$. The value of the secondary deformation modulus $\mathrm{E}_{2}=75 \mathrm{MPa}$ should be described as sufficient, because the CBR index for this aggregate $(\mathrm{CBR}=57.2 \%$ - Tab. 2) suggests obtaining a relatively high stiffness for this layer. Similarly, when analysing the grain size of burned shale, inscribed in the field of proper graining for all surface layers, i.e. layers of improved subgrade, subbase and base course (Fig. 2), a high stiffness of this layer, higher than $100 \mathrm{MPa}$, is expected. Obtaining a modulus lower than expected should be combined with the stiffness of the lower layer, i.e. coarse sand, which is a uniformly-graded material, i.e. hardly compactable.

The results of cyclic loading of the layer system allow to observe a rapid increase of the vertical displacement (subsidence of the plate) in the initial cycles, and then slow stabilization of vertical displacements (Fig. 7) between subsequent load cycles. The vertical displacement of the plate, which decreases in subsequent cycles, indicates that the tested medium densifies and increases its stiffness (load-bearing capacity). It should be noted, however, that in cyclic tests the modulus began to stabilize at around $70 \mathrm{MPa}$. A similar value of the deformation modulus E2 $=80 \mathrm{MPa}$ was obtained during the last (stage III) static test with the VSS plate. The obtained value of the modulus, at high density $\mathrm{I}_{0}=1.78$, indicates a relatively small increase in stiffness of the upper layer, compared to the test carried out before the cyclic tests. This increase is definitely lower than expected. The unfavourable parameters of the sand layer constituting the subgrade of the examined shale should be considered as the probable cause of the relatively small increase of the secondary deformation modulus as well as the obtained value of the secondary deformation modulus whose value was lower than expected. These observations are also confirmed by the preliminary results of numerical analyses (Fig. 8), consisting in the identification of the parameters of the adopted Mohr-Coulomb model. The iterative method was used to find the parameter values, striving for the best fit of the theoretical curve (numerical calculations) presenting the displacement $[\mathrm{mm}]$ as a function of the load $[\mathrm{kPa}]$, to the experimental curve 
(from the VSS plate measurement). The performed back calculations indicate a low sand modulus (about $20 \mathrm{MPa}$ ) and a definitely higher value of the aggregate layer modulus (about $85 \mathrm{MPa}$ ) (Tab.4). Taking into account such significant differences in resilient moduli, the properties of burned shale should be positively evaluated, because, as the tests indicate, the modulus of resilience of unbound layers is strongly determined by the stiffness of the base on which the subject layer is embedded. The results of numerical analyses also indicate one more phenomenon determining the value of the deformation modulus. This phenomenon is associated with displacing the material from under the load plate (Fig. 9), which causes an increase in the vertical displacement and reduction of the value of the modulus.
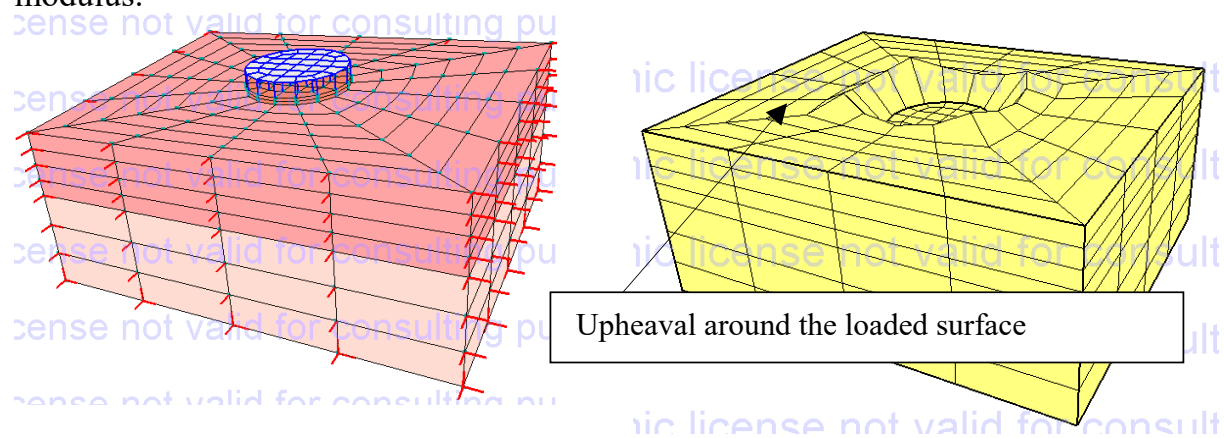

Fig. 8. Calculation model\#

Fig. 9. Model deformations and calculated displacements\#

Table 4. Parameters of the Mohr - Coulomb model

\begin{tabular}{|c|c|c|c|c|c|c|}
\hline \multirow{2}{*}{ Layer } & \multicolumn{7}{|c|}{ Iteration - 21.04 } \\
\cline { 2 - 7 } & $\mathbf{E}[\mathbf{M P a}]$ & $\mathbf{c}[\mathbf{k P a}]$ & $\boldsymbol{\varphi}\left[^{\circ}\right]$ & $\boldsymbol{\Psi}\left[^{\circ}\right]$ & $\boldsymbol{v}$ & $\gamma\left[\mathbf{k N} / \mathbf{m}^{\mathbf{3}}\right]$ \\
\hline Steel plate $2 \mathrm{~cm}$ & 210,000 & - & - & - & 0.20 & 75 \\
\hline Burned shale $20 \mathrm{~cm}$ & 85 & 2 & 45 & 15 & 0.35 & 17 \\
\hline Sand $30 \mathrm{~cm}$ & 20 & 2 & 39 & 9 & 0.35 & 17 \\
\hline
\end{tabular}

The fact that the grains of burned shale during the cyclic tests were broken (fragmentation) is not without significance for shaping the stiffness of the layer. This phenomenon is indicated by the experimental field studies described by Rafalski [8]. Therefore, this observation is significant as the grain curve of grain size of burned shale before the cyclic tests was carried out in close proximity to the upper curve of proper graining (Fig. 2 and Tab. 2).

\section{Conclusion}

The conducted tests using the station for cyclic loads of the system of unbound layers allowed in real conditions to assess the impact of repetitive loads on the mechanical properties of the tested layers. Such tests provide the opportunity to assess the behaviour of the layering system in conditions similar to those that occur during their use under a functional load, i.e. in conditions such as loading the pavement with circular motion.

The obtained results of the research well describe the causal relationship between the material properties of individual layers and the results obtained on the surface of this layer (deformation modulus). This type of testing station can be a tool for verification of other material solutions as well as it can be used to assess the impact of other phenomena occurring in the ground subgrade and/or in the pavement layers on its stiffness and 
indirectly on its fatigue durability. The assessment of burned shale carried out on the basis of material tests (e.g. CBR, graining), cyclic loading and preliminary numerical analyses, it should be stated that this material meets the requirements for the frost protection layer and the lower layers of the pavement and layers of the improved subgrade. The results of the tests performed by other researchers, as well as the analysis of the results of own research, indicate the susceptibility of this material to fragmentation, which may affect the lower than expected increase in stiffness of the layer. In terms of the use of burned shale in the lower layers of the pavement, it seems justified to perform tests taking that would include variable moisture of the material, caused for example by periodic exposure to water, which impacts on the durability of road pavements [10]. Using the testing station will be also useful to evaluate the interaction between the geogrid and the unbound aggregate, which would broaden the research described by Grygierek and Kawalec [11].

\section{References}

1. D.J. White, K.R. Vennapusa Pavana, In situ resilient modulus for geogrid-stabilized aggregate layer: A case study using automated plate load testing, Transportation Geotechnics, 11, 120-132 (2017) http://dx.doi.org/10.1016/j.trgeo.2017.06.001

2. A. Hustvig, A new mechanistic model for prediction of permanent strain in unbound friction materials DOI: 10.13140/RG.2.1.4371.7528

3. J. Pieczyrak, Właściwości fizyczne oraz skład chemiczny i mineralny przepalonych odpadów kopalnianych (Physical Properties as well as Chemical and Mineral Composition of Burned Mining Waste) (in Polish), OTG, 62, 30-38 (1982)

4. Y. Qian, J. Han, S.K. Pokharel, R.L. Parsons, Determination of Resilient Modulus of Subgrade Using Cyclic Plate Loading Tests, Geo-Frontiers, ASCE, 4743-4751 (2011)

5. X. Sun, J. Han, J. Kwon, R.L. Rarsons, M.H. Wayne, Radial stresses and resilient deformations of geogrid-stabilized unpaved roads under cyclic plate loading tests, Geotextiles and Geomembrans, 43, 440-449 (2015)

6. WT-4, Unbound mixes for national roads, Technical Requirements. General Directorate of National Roads and Motorways (in Polish) (2010)

7. J. Pieczyrak, Characteristics and engineering usage of waste from coal mining, ACEE, 1 (2010)

8. L. Rafalski, Road base-courses (in Polish), Issue Studies and materials, 59 (2007)

9. Catalogue 2014-2, Catalogue of typical flexible and semi-rigid pavement structures. General Directorate of National Roads and Motorways (in Polish) (2014)

10. P. Rokitowski, M. Grygierek, Influence of high water contents on pavement layers stiffness caused by flooding, Civil Engineering Series, 16, 1 (2016) DOI 10.1515/tvsb2016-0007

11. M. Grygierek, J. Kawalec, Selected Laboratory Research on Geogrid Impact on Stabilization of Unbound Aggregate Layer, Procedia Engineering, 189, 484-491 (2017) DOI: $10.1016 /$ j.proeng.2017.05.078 